\title{
MAGNETS FOR A MUON STORAGE RING*
}

\author{
B. Parker , M. Anerella, J. Escallier, A. Ghosh, R. Gupta, M. Harrison, \\ J. Schmalzle, J. Sondericker and E. Willen, BNL, Upton, NY 11973, USA
}

\begin{abstract}
We present a new racetrack coil magnet design, with an open midplane gap, that keeps decay particles in a neutrino factory muon storage ring from directly hitting superconducting coils [1]. The structure is very compact because coil ends overlap middle sections top and bottom for skew focusing optics [2]. A large racetrack coil bend radius allows "react and wind" magnet technology to be used for brittle $\mathrm{Nb}_{3} \mathrm{Sn}$ superconductors.

We describe two versions: Design-A, a magnet presently under construction and Design-B, a further iterated concept that achieves the higher magnetic field quality specified in the neutrino factory feasibility StudyII report [1]. For Design-B reverse polarity and identical end design of consecutive long and short coils offers theoretically perfect magnet end field error cancellation. These designs avoid the dead space penalty from coil ends and interconnect regions (a large fraction in machines with short length but large aperture magnets) and provide continuous bending or focussing without interruption. The coil support structure and cryostat are carefuly optimized.
\end{abstract}

\section{INTRODUCTION}

Muons circulating in a neutrino factory storage ring decay to produce both neutrinos that go to distant experiments and other particles that shower and deposit energy inside the ring. An important goal for both the neutrino factory and higher energy muon collider magnet designs is to minimize the deposited energy in the superconductor. The proposed design has racetrack coils and open midplane, as shown in Fig. 1, to keep the coils away from decay particles and has no "tungsten liner."

Performance, cost and environmental considerations require the storage ring size be minimized. At a BNL site environmental impact considerations are especially crucial. The ring is tilted 13.1 degrees from horizontal and the lower end must be kept above the water table (Long Island's drinking water source) The water table is as close as $\approx 11$ meters below ground level. Thus much of the ring would be above ground level and machine size determines the additional amount of hill that has to be constructed.

\section{MAGNET DESIGN}

We start with an initial magnet design condition that the superconducting collared coils inside a cryostat clear the magnet midplane region where most of the decay energy goes. A warm iron yoke structure around the coils then allows heat generated by decay particles to be removed efficiently at higher temperature. Though optimized for

\footnotetext{
*Work supported in part by U.S. Department of Energy under contract No. DE-AC02-98CH10886 (BNL)
}

parker@bnl.gov, web page is at http://magnets.bnl.gov/Staff/Parker/

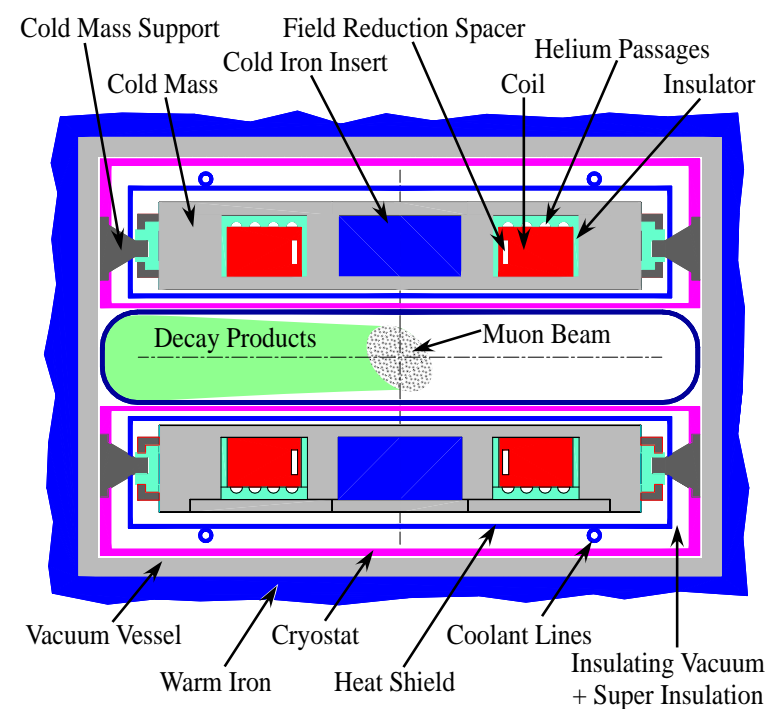

Figure 1: Cross section, with main features labeled, of neutrino factory muon storage ring magnet that avoids decay particles directly hitting superconducting coils. present requirements, many design principles can be extended to higher energy, and higher field, for a muon collider magnet and/or any magnet that must deal with large amounts of energy deposited near the midplane.

The dipole operating field is $6.93 \mathrm{~T}$ and the quench field is over $8 \mathrm{~T}$ for an operating field margin of over $15 \%$. The maximum field on the conductor at quench is significantly higher than the central field and excludes using $\mathrm{NbTi}$ at $4.2 \mathrm{~K}$. The coils therefore are made from brittle $\mathrm{Nb}_{3} \mathrm{Sn}$ superconductor. A large end bend radius allows using "react and wind" in a pancake coil (racetrack) geometry. Both Design-A, the magnet under construction, and Design-B, the current feasibility Study-II design, use $\mathrm{Nb}_{3}$ Sn with "react and wind" technology.

\subsection{Design-A Coil Configuration}

Significant design work was already completed while Design- $B$ was still evolving so Design- $A$ is the magnet under construction. It is a proof-of-principle magnet being built with modest resources from the Laboratory Directed Research and Development (LDRD) program and uses free, but lower performance, $\mathrm{Nb}_{3} \mathrm{Sn}$ conductor. It gives a first step towards demonstrating this magnet design and technology although only $\approx 4 \mathrm{~T}$ field will be produced with this conductor rather than the desired $8 \mathrm{~T}$.

The Design-A configuration, shown in Fig. 2, uses the same coil in cryostat package above and below the beam. The coil packages are longitudinally staggered to provide $\mathrm{F}$ or D skew quadrupole combined function focusing where there is only a single coil top or bottom. A pure dipole field occurs in overlap regions. The dipole guide field in non-overlap regions is about half that in overlap 


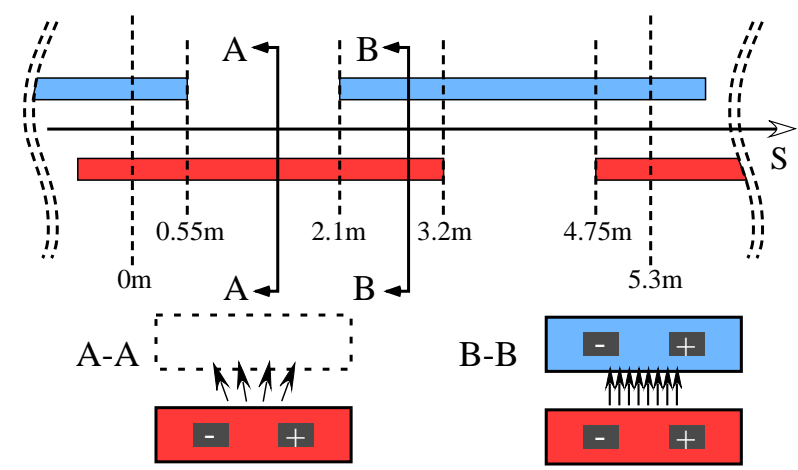

Figure 2: Design-A coil and cryostat layout schematic. Regions with no coil overlap, A-A, have half strength dipole field + skew quadrupole field. Full dipole field and no skew quadrupole occurs in overlap region, B-B.

regions. Thus, the structure has continuous bending and alternate gradient skew focusing. Space normally lost at coil ends and magnet interconnects is efficiently used.

Since the muon storage ring magnets operate at fixed excitation, optimizing the Design-A coil two dimensional cross section for good body field harmonics is straight forward. We adjust coil position, coil cross section (a spacer performs the same function as a $\operatorname{Cos}(\theta)$ coil design wedge), and a magnetic insert located in the cold mass pole region. The coils are wound in double pancake layers to avoid having lead connections in the pole. Rectangular spacers reduce coil peak field and improve field quality.

During coil design challenging end field harmonic targets were proposed and major changes to the coil ends (stretching) would have been needed. Instead we adopt a second coil layout, that takes advantage of Design-A gaps to insert a short reverse polarity coil powered with current going in the opposite direction as in the longer main coils.

\subsection{Design-B Coil Configuration}

The Design- $B$ configuration with short reverse polarity coils in gaps between main coils is shown in Figs. 3 and 4. In the reverse coil region the dipole field goes to zero and the skew quadrupole field is a factor of two more compared to Design-A. Both designs have nearly the same arc size. The Design- $B$ bending is not continuous but the skew quadrupole field is stronger and the skew region is shorter so more cell length is available for dipole bending.

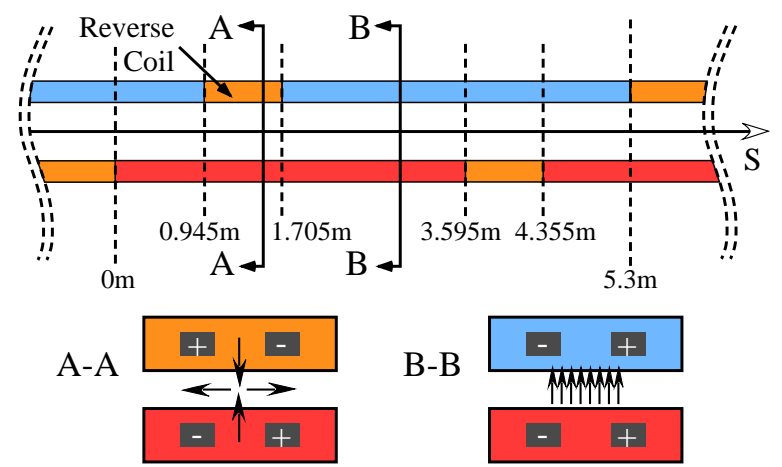

Figure 3: Design-B coil and cryostat layout schematic. Opposite polarity regions, A-A, have skew quadrupole field and same polarity, B-B, have the full dipole field.

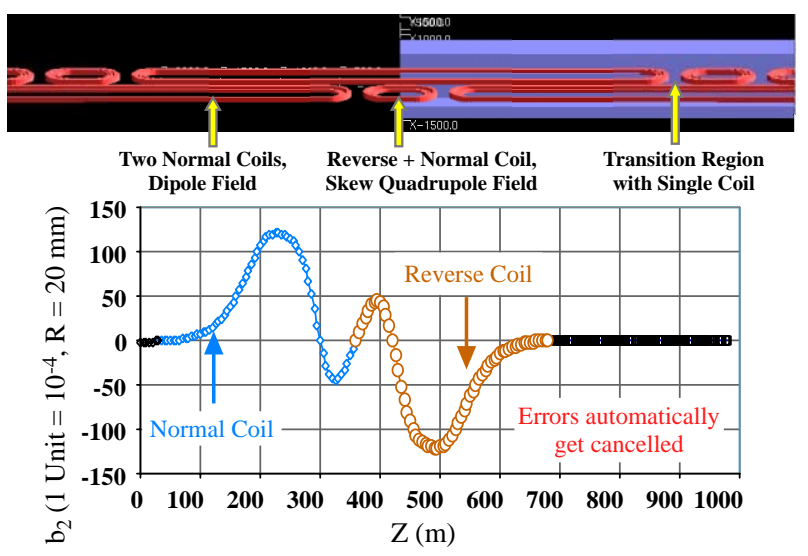

Figure 4: Design-B coil layout and an example of end harmonic cancellation. Since normal and reverse coils have same ends, their coil end field harmonics cancel.

The major benefit which comes with this configuration is the near automatic end field harmonic cancellation shown in Fig. 4. The cancellation comes from symmetry and not from stretching out coil ends. The expected field harmonic errors for the dipole and skew quadrupole sections, for a theoretical magnet design in combination with previous magnet design and construction experience, are given in Tables 1 and 2. Quite likely some of the harmonics listed will have to change in order to balance other magnet design and construction related issues. Baseline magnetic field calculations for many coil

Table 1: The estimated Design- $B$ dipole field errors at $20 \mathrm{~mm}$ reference radius, $b_{0}=10^{4} .\left\langle b_{n}\right\rangle$ and $\left\langle a_{n}\right\rangle$ are expected means of normal and skew terms, $\delta\left(b_{n}\right)$ and $\delta\left(a_{n}\right)$ systematic uncertainties, and $\sigma\left(b_{n}\right)$ and $\sigma\left(a_{n}\right)$ random uncertainties. $n=2$ corresponds to a sextupole.

\begin{tabular}{|c|ccc|ccc|}
\hline$n$ & $\left\langle b_{n}\right\rangle$ & $\delta\left(b_{n}\right)$ & $\sigma\left(b_{n}\right)$ & $\left\langle a_{n}\right\rangle$ & $\delta\left(a_{n}\right)$ & $\sigma\left(a_{n}\right)$ \\
\hline $\mathbf{1}$ & 0 & 0.2 & 0.2 & 0 & 1 & 2 \\
$\mathbf{2}$ & -1 & 1 & 2 & 0 & 0.1 & 0.5 \\
$\mathbf{3}$ & 0 & 0.1 & 0.1 & 0 & 0.3 & 1 \\
$\mathbf{4}$ & -1 & 1 & 1 & 0 & 0.05 & 0.2 \\
$\mathbf{5}$ & 0 & 0.03 & 0.03 & 0 & 0.1 & 0.5 \\
$\mathbf{6}$ & -0.3 & 0.2 & 0.1 & 0 & 0.03 & 0.1 \\
$\mathbf{7}$ & 0 & 0.03 & 0.01 & 0 & 0.03 & 0.1 \\
$\mathbf{8}$ & -0.1 & 0.1 & 0.02 & 0 & 0.03 & 0.1 \\
$\mathbf{9}$ & 0 & 0.03 & 0.01 & 0 & 0.03 & 0.1 \\
$\mathbf{1 0}$ & -0.03 & 0.02 & 0.02 & 0 & 0.03 & 0.1 \\
\hline
\end{tabular}

Table 2: The estimated field Design-B skew quadrupole errors, $a_{1}=10^{4}$. See Table 1 for definition of terms.

\begin{tabular}{|c|ccc|ccc|}
\hline$n$ & $\left\langle b_{n}\right\rangle$ & $\delta\left(b_{n}\right)$ & $\sigma\left(b_{n}\right)$ & $\left\langle a_{n}\right\rangle$ & $\delta\left(a_{n}\right)$ & $\sigma\left(a_{n}\right)$ \\
\hline $\mathbf{1}$ & 0 & 0.2 & 0.2 & - & 1 & 2 \\
$\mathbf{2}$ & -0.5 & 0.5 & 1 & 0 & 1 & 0.5 \\
$\mathbf{3}$ & 0 & 0.1 & 0.1 & 2 & 2 & 1 \\
$\mathbf{4}$ & -0.5 & 0.5 & 0.5 & 0 & 0.05 & 0.2 \\
$\mathbf{5}$ & 0 & 0.03 & 0.03 & 1 & 1 & 2 \\
$\mathbf{6}$ & 0 & 0.2 & 0.1 & 0 & 0.03 & 0.1 \\
$\mathbf{7}$ & 0 & 0.03 & 0.01 & 0.5 & 0.5 & 0.3 \\
$\mathbf{8}$ & 0 & 0.1 & 0.05 & 0 & 0.03 & 0.1 \\
$\mathbf{9}$ & 0 & 0.03 & 0.01 & 0.1 & 0.03 & 0.1 \\
$\mathbf{1 0}$ & 0 & 0.02 & 0.01 & 0 & 0.03 & 0.1 \\
\hline
\end{tabular}




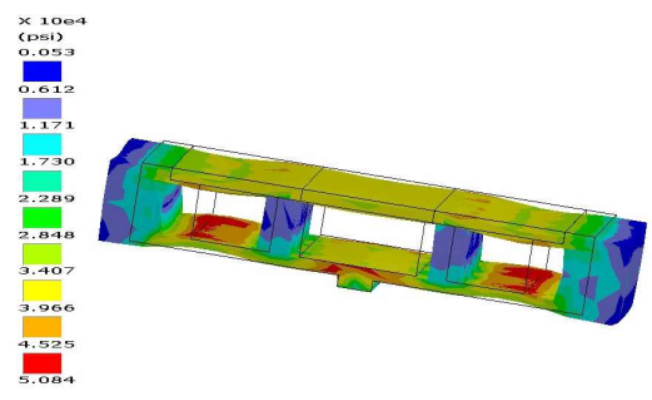

Figure 5: Finite element calculation of the stress in the cold mass, in of units $10^{4} \mathrm{psi}(10 \mathrm{kpsi}=70 \mathrm{MPa})$, due the $18 \mathrm{kpsi}$ outward coil force found at $8 \mathrm{~T}$ quench field.

configurations were used to support these numbers. Reducing field harmonics has pushed the magnet design toward a parameter space with larger coil forces. If it is found acceptable to slightly relax some of the harmonic goals, then the magnet design could be further simplified.

\subsection{Mechanical Design Considerations}

The present design has the iron yoke and beam tube warm but the coils are contained in cold masses surrounded by a heat shield and cryostat. To maintain field strength, we minimize the vertical distance between the coils and the beam cavity. The cryostat wall thickness is minimized on the side near the beam tube. Surrounding both cold masses and the beam cavity is an outer vacuum vessel that eliminates differential pressure on the cryostats and beam tubes and prevents them from collapsing under vacuum. The beam cavity wall is as thin as possible.

At the maximum quench field of $8 \mathrm{~T}$ the coils generate considerable forces. The maximum forces are $\approx 18 \mathrm{kpsi}$ outward and $\approx 4 \mathrm{kpsi}$ vertically. The cold mass structure is designed to withstand the outward coil forces. Fig. 5 shows output from finite element analysis of the stress in the cold mass coil support structure.

The beam cavity size (large to contain decay particles) and vertical spacing budget (as small as possible to maximize transfer function) make it necessary to rely on support keys that mount on the sides of the cold mass to handle vertical attractive/repulsive forces. The supports mount to the cryostat, which is itself supported by the vacuum vessel. Numerous keys are needed to support the coil loads and these keys need to be quite massive. They therefore represent a substantial heat load.

The keys have been designed with tapered edges to reduce their size. To further decrease heat load, the key engages in an insulator mounted to the cold mass and only makes contact with the insulator on one side. Forces vary both in magnitude and direction at different longitudinal positions in the magnet because of coil polarity flips and the upper and lower coil being offset. So we optimize the support orientation and frequency to ensure that there is sufficient local support while minimizing heat load.

\subsection{Magnet Production and Testing}

Our LDRD test magnet design has several racetrack coils, in independent cryostats, surrounded by a warm

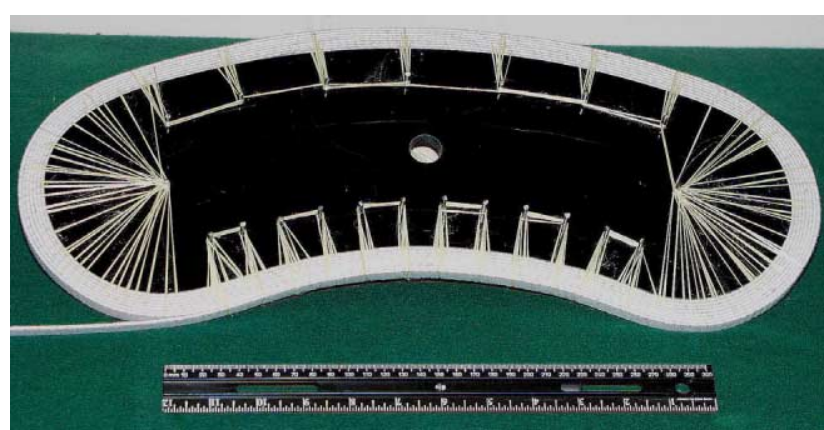

Figure 6: Winding technique for coils with reverse bends.

iron yoke as in Fig. 1, and the same length as a Design-B short reverse polarity coil. When these coils are powered with the same polarity a dipole field results and with opposite polarity a skew quadrupole field results. Finally by shifting the two cryostats in opposite longitudinal directions we can test coil end transitions.

Within a cryostat the coil package consists of two double pancake layers (total of four layers). Though made from ITER $\mathrm{Nb}_{3} \mathrm{Sn}$ strand conductor, the cable geometry is similar to a $1 \mathrm{~cm}$ wide RHIC style cable. The coils will be wound and vacuum impregnated using techniques developed for the BNL common coil magnet program [3].

A special challenge in making bona fide muon storage ring magnet coils is that they have considerable saggitta. We intend to develop further the winding technique shown in Fig. 6 for constructing coils with reverse bends. During winding the conductor is held temporarily in place by fibers under tension. We anticipate winding and testing reverse bend coils made from NbTi before applying the technique to brittle materials such as $\mathrm{Nb}_{3} \mathrm{Sn}$ and high temperature superconductor (HTS).

\section{SUMMARY}

Superconducting magnets for the muon storage ring of the Study II neutrino factory must be compatible with building compact arcs and should tolerate large internal energy deposition from decay particles. We now have a design for a new type of magnet system that meets both goals: the first through overlap of long and short flat pancake coils thereby creating a space efficient skew focusing lattice structure and the second by keeping the top and bottom coils apart and away from the beam in cryostats supported inside a common warm yoke. We are embarking on a program of magnet production and testing along these lines that is quite favorably leveraged by the present common coil program activities at BNL.

\section{REFERENCES}

[1] S. Ozaki, et.al., "Neutrino Factory and Muon Collider Collaboration Feasibility Study II Report," http://www.cap.bnl.gov/mumu/studyii/FS2-report.html, June, 2001.

[2] B. Parker, "Skew Quadrupole Focusing Lattices and Applications," paper MOPA010 this conference.

[3] J. Escallier, et.al., "Technology Development for React and Wind Common Coils," paper TOAB011 this conference. 